\title{
Evaluación auténtica como alternativa para la mejora en el proceso de enseñanza-aprendizaje de educación física en escuelas primarias
}

\author{
Authentic evaluation: an alternative to improve the teaching- \\ learning process in primary school physical education
}

${ }^{*}$ Eversley Bernard George \& * Patricia Adelaida González-Moreno

\begin{abstract}
George, E. B., \& González-Moreno, P. A. (2020). Evaluación auténtica como alternativa para la mejora en el proceso de enseñanza-aprendizaje de educación física en escuelas primarias. Revista Ciencias de la Actividad Física UCM, N²1(2), juliodiciembre, 1-19. DOI: http://doi.org/10.29035/rcaf.21.2.3
\end{abstract}

\section{RESUMEN}

El objetivo de esta investigación fue examinar las estrategias, instrumentos y criterios de evaluación auténtica que aplican los maestros de educación física en nivel primaria, en función de la planificación y la aplicación de secuencias didácticas previamente establecidas para su proceso de intervención en el aula. Participaron voluntariamente tres maestros de educación física de tres diferentes escuelas, una escuela federal, una estatal y una privada en el estado de Chihuahua, México. El trabajo de investigación se caracterizó como un estudio de casos múltiple donde los maestros participaron en un taller de evaluación auténtica y elaboraron secuencias didácticas que fueron implementadas para examinar la aplicación de estrategias e instrumentos de evaluación auténtica. El análisis cualitativo de las observaciones, cuestionarios y entrevistas sugieren que la aplicación de dichas secuencias didácticas, junto con la implementación de una evaluación auténtica, favoreció el desempeño docente y enriqueció el ambiente de aprendizaje. Las implicaciones del estudio apuntan hacia la necesidad de formalizar y sistematizar la aplicación de estrategias e instrumentos de evaluación auténtica en la educación básica, cuando estos están directamente relacionados con las secuencias didácticas y los contenidos curriculares.

Palabras clave: evaluación, evaluación auténtica, educación física, secuencias didácticas, educación primaria.

\section{ABSTRACT}

The aim of this study was to examine the strategies, instruments, and criteria of authentic evaluation that physical education teachers apply at the primary level, based on the planning and application of didactic sequences previously established for an intervention process. Initially and voluntarily, three physical education teachers participated from three different schools, one federal, one state, and one private, in Chihuahua State, Mexico. The research project was characterized as a multiple case study, where teachers participated in an authentic evaluation workshop and developed didactic sequences that were implemented to examine the application of strategies and instruments of authentic evaluation. The qualitative analysis of the observations, questionnaires, and interviews suggest that the application of these didactic sequences together with the use of authentic evaluation strategies, favored teaching performance and enriched the learning environment. The implications of the study point to the need to formalize and systematize the application of authentic evaluation strategies and instruments within basic education when these are causally related to the didactic sequences and the curriculum content.

Key words: evaluation, authentic evaluation, physical education, didactic sequence, primary education. 
George, E. B., \& González-Moreno, P. A. (2020). Evaluación auténtica como alternativa para la mejora en el proceso de enseñanza-aprendizaje de educación física en escuelas primarias. Revista Ciencias de la Actividad Física UCM, № 21(2), julio-diciembre, 1-19.

DOI: http://doi.org/10.29035/rcaf.21.2.3

\section{INTRODUCCIÓN AL ESTUDIO}

La brecha entre lo que los maestros dicen que quieren hacer y lo que están haciendo, en la práctica ha sido y sigue siendo uno de los problemas que afectan a la educación física, como lo es en muchas ramas de la educación (Osborne, Belmont, Peixoto, Azevedo \& Carvalho, 2016). En recientes años se han modificado los planes de estudio en México por lo que la educación física, también encuentra cambios que apuntan a un perfil docente apoyado y valorado de acuerdo con lo establecido en dichos currículos (Ramírez \& Chel, 2019, SEP, 2019). No obstante, de lo escrito en el papel a la práctica aún existe una separación. Estos planes son aplicados en el estado de Chihuahua, y aunque enumeran diferentes tipos de métodos de enseñanza y evaluación, no está claro el aspecto pedagógico en los docentes. Aunque se puede encontrar un excelente análisis de los objetivos de la educación física en estos recursos, así como instrucciones detalladas sobre cómo enseñar diferentes tipos de actividades, falta el tema más importante: una evaluación que refleje la situación real de aprendizaje de los estudiantes. Esto es motivo de preocupación, por tanto, es necesario desarrollar estrategias e instrumentos de evaluación que sean reales y auténticos para mejorar el proceso de enseñanza-aprendizaje.

Uno de los procesos más desafiantes en educación es la evaluación. Esto puede ser invaluable para ayudar a los maestros a identificar las fortalezas, debilidades y autoevaluarse de cada estudiante y guiar el siguiente paso hacia su educación. Pero ¿qué queremos decir realmente cuando evaluamos? Según Mertens (2015), el propósito general de la evaluación es crear conciencia sobre la necesidad de comprender mejor el concepto de facilitación y, por lo tanto, mejorar su operacionalización deliberativa. Es decir, hacer que el alumno se dé cuenta de dónde hay y qué debe hacer para mejorar.

Cuando se trata de los diferentes tipos de evaluación en educación, la mayoría de los trabajos publicados en las últimas dos décadas siguen haciendo referencia a los conceptos más clásicos, por ejemplo: evaluación formal, evaluación informal, evaluación continua, evaluación diagnóstica, evaluación formativa, evaluación sumativa, evaluación cuantitativa, evaluación cualitativa, evaluación tradicional o evaluación por competencias (López-Pastor \& Pérez-Pueyo, 2017). Ha habido un cambio en la literatura que reconoce nuevos tipos de evaluación en educación y es notablemente interesante desde un punto de vista pedagógico: evaluación auténtica, evaluación para aprendizaje, evaluación alternativa, etc. El concepto de evaluación auténtica se utiliza con una frecuencia mayor (Bazmi \& Ahmed, 2018, Brown, 2015, Georgakis, Wilson \& Evans, 2015, Gulikers, Bastiaens \& Kirschner, 2004, Hay \& Penney, 2013, Moreno, 2012, Mintah, 2003), en referencia a las técnicas, instrumentos, estrategias y actividades de evaluación que se aplican claramente en la saturación, las actividades y el contenido que es real en el proceso de aprendizaje.

\section{Planteamiento del Problema}

En México, a pesar de los programas de educación física desarrollados por el Instituto Nacional de Salud Pública (INSP, 2018) y avalados por la UNESCO, la carga horaria semanal de esta disciplina en educación básica (SEP, 2017) pone en evidencia la falta de apoyo institucional e inconsistencias entre el discurso político sobre la importancia y necesidad de la actividad física en los estudiantes y la realidad educativa. Además, aunque el actual currículo y sus "Aprendizajes Clave" (SEP, 2017) contemplan una educación física de calidad e inclusiva, es muy débil en cuanto a la forma de 
George, E. B., \& González-Moreno, P. A. (2020). Evaluación auténtica como alternativa para la mejora en el proceso de enseñanza-aprendizaje de educación física en escuelas primarias. Revista Ciencias de la Actividad Física UCM, № 21(2), julio-diciembre, 1-19.

DOI: http://doi.org/10.29035/rcaf.21.2.3

evaluación que debe emplear el docente, para asegurar que los objetivos curriculares sean alcanzados. Las clases van encaminadas al desarrollo de habilidades deportivas y en menor medida se la ha considerado como un área orientada al fomento de la salud y del desarrollo integral de todos los estudiantes (LópezTaylor, Jáuregui-Ulloa \& González-Villalobos, 2013).

Según el informe realizado por el INSP (2018), la Secretaría de Educación Pública cuenta con cerca de 90,000 maestros de Educación Física para los 255,000 planteles, por lo que una gran proporción de las sesiones son impartidas por maestros generales y no especialistas en la materia. Solo el 35\% de estos maestros están especializados para enseñar en esta área (INSP, 2018, p. 42). Esto sugiere que la carencia de profesionales en el área puede influenciar negativamente en el desempeño de los estudiantes y la calidad con la que reciben los contenidos de dicha disciplina. Sumado a lo anterior, el INSP (2018), reconoce que:

"respecto a las áreas de conocimiento de los planes y programas, el estudio denotó una ausencia del área pedagógica, reportando que las más predominantes eran el área biológica, el entrenamiento deportivo, la salud/nutrición/calidad de vida y la recreación, mientras que el área psicopedagógica, de gestión/administración y de investigación aplicada a la educación física y/o deporte eran las menos presentes" (p. 37).

Esto sugiere que muchos de los profesionales en el área, podrían no contar con las competencias necesarias para la utilización de diferentes métodos y/o estrategias de enseñanza y aún más de evaluación. A esto hay que agregar que los recursos y materiales didácticos que faciliten la impartición y evaluación de la clase, en algunos casos, son muy escasos. Es decir, que el sistema de educación física en México es muy débil en el proceso de evaluación y no hay una evaluación disponible para determinar el éxito o el fracaso en el logro de los desempeños esperados (López-Taylor, Jáuregui-Ulloa \& González-Villalobos, 2013).

Asimismo, la forma de evaluación es una de las desventajas que tradicionalmente han caracterizado la educación física en el estado de Chihuahua. No obstante, el INSP (2018) señala, además, que hay espacio para desarrollar la asignatura de la educación física con enfoques pedagógicos específicos y estrategias para evaluar la evolución de los alumnos, distintas de las empleadas para valorar otros campos formativos como español y matemáticas. Por esta razón, es imperativo que los educadores conozcan e implementen medios y estrategias alternativas de evaluación, que muestren una mayor validez y confiabilidad para examinar el desempeño en el proceso de enseñanza-aprendizaje.

En este sentido, la evaluación auténtica abre nuevas perspectivas a una de las tareas docentes más complejas y difíciles de desarrollar, como lo es la evaluación de los aprendizajes de los alumnos (Vallejo \& Molina, 2014). La evaluación auténtica es un proceso que incluye cuatro componentes básicos, como son: medir la mejora a lo largo del tiempo, motivar a los alumnos a estudiar, evaluar los métodos de enseñanza y la clasificación de las capacidades de los alumnos en relación con la evaluación de todo el grupo (Jabbarifar, 2009). Estos componentes apuntan al rol del docente en los procesos de enseñanza y aprendizaje, no solo respecto al dominio de los contenidos curriculares, sino también de otras áreas complementarias para poder conducir a sus alumnos hacia las metas preestablecidas por los planes y programas de educación física, tomando como base la evaluación auténtica. Este tipo 
George, E. B., \& González-Moreno, P. A. (2020). Evaluación auténtica como alternativa para la mejora en el proceso de enseñanza-aprendizaje de educación física en escuelas primarias. Revista Ciencias de la Actividad Física UCM, N²1(2), julio-diciembre, 1-19.

DOI: http://doi.org/10.29035/rcaf.21.2.3

de evaluación, según Hay \& Penney (2009) y Mintah (2003), es más objetiva y justa para valorar los procesos de aprendizaje, pues utiliza diversas formas de medir el desempeño de los estudiantes. Este autor menciona algunos tipos de evaluación auténtica como son las entrevistas orales, la narración de la historia o del texto, ejemplos de tipos de escritura, proyectos y/o exhibiciones, experimentos/demostraciones, pruebas o exámenes, observaciones del maestro, portafolios y videos de evaluación auténtica, entre otros.

La evaluación auténtica ofrece una oportunidad para que los estudiantes realicen tareas auténticas que sean interesantes, útiles y relevantes para su vida. Esta tarea puede hacer que estos sean innovadores y creativos porque tienen la oportunidad de desarrollarse, fomentar una actitud más positiva hacia la escuela y las actividades de aprendizaje. Está basada en la reflexión de las enseñanzas, los aprendizajes, los logros y la motivación, tanto de los maestros como de los alumnos. Aprender de manera significativa es la base de la evaluación auténtica, pues el aprendizaje y competencias que adquiera el estudiante podrán ser usados en la vida cotidiana (Karim, Abduh, Manda \& Yunus, 2018).

Para que la evaluación auténtica cumpla con su cometido, es prioritario que exista una conexión entre la experiencia educativa y los ámbitos personal, profesional y social del individuo. Los estudiantes que realizan evaluaciones significativas y relevantes tienden a participar más plenamente en el aprendizaje, lo cual está significativamente relacionado con el logro de objetivos más altos, pues lo que están haciendo tiene sentido. Así, la evaluación auténtica es un claro ejemplo de dicho tipo de evaluaciones, ya que esta desafía a los estudiantes, quienes a menudo no se han encontrado con actividades de evaluación en las cuales se les pide pensar de manera creativa (Brown, 2015).

Cabe señalar que la educación física afecta en gran medida el estilo de vida y la salud de las personas (Molina-Luque, 2018), por lo tanto, debe tenerse en cuenta su relevancia en la infancia, así como en todas las etapas de la vida, y sus beneficios una vez que se canaliza correctamente. Por lo tanto, la educación física debe considerarse prioritaria dentro del plan de estudios en educación básica e introducir una evaluación que sea real y efectiva en el proceso de enseñanza y aprendizaje de cada alumno.

La falta de una evaluación que refleje la situación real de aprendizaje de los estudiantes es un motivo de preocupación, por lo que se hace necesario desarrollar estrategias e instrumentos de evaluación auténtica para mejorar el proceso de enseñanza-aprendizaje, incrementar la motivación intrínseca del estudiante de manera que afecte positivamente su desempeño, tanto académico, como su interés de participar en la clase de educación física, así como aumentar su intención de practicar actividad físico-deportiva fuera del contexto escolar.

\section{Objetivo y Preguntas de Investigación}

El objetivo de esta investigación fue examinar las estrategias, instrumentos y criterios de evaluación auténtica que aplican los maestros de educación física en nivel primaria, en función de la planificación y la aplicación de secuencias didácticas previamente establecidas para su proceso de intervención en el aula. Con base en este objetivo, se plantearon las siguientes preguntas de investigación:

- ¿Cuáles son los tipos de evaluación usualmente utilizados para medir los aprendizajes en educación física en el nivel primaria? 
George, E. B., \& González-Moreno, P. A. (2020). Evaluación auténtica como alternativa para la mejora en el proceso de enseñanza-aprendizaje de educación física en escuelas primarias. Revista Ciencias de la Actividad Física UCM, № 21(2), julio-diciembre, 1-19.

DOI: http://doi.org/10.29035/rcaf.21.2.3

- ¿En qué medida un programa de formación que integre estrategias de evaluación auténtica facilita cambios en la forma de evaluar de los maestros de educación física?

- ¿Cuáles son los recursos de evaluación auténtica y cómo son utilizados por los maestros(as) en la clase de educación física para evaluar el desempeño estudiantil, después de haber recibido capacitación sobre el tema de secuencias didácticas y procesos de evaluación auténtica?

- ¿Cuál es la valoración del proceso de evaIuación auténtica comparándolo con la planificación didáctica de las clases de educación física?

\section{Método}

Este trabajo adopta el enfoque metodológico de investigación cualitativa. Se consideró el empleo de un diseño de investigación de estudio de casos múltiples, pues de acuerdo con Yin (2018) con este se obtienen elementos de interés desde una visión particular, compleja. Además, de la capacidad de este para obtener información exhaustiva de cada caso.

\section{Participantes}

Para este estudio se utilizó el muestreo por conveniencia en función de la disponibilidad y experiencia docente de los participantes, lo que permitió a los investigadores localizar a individuos que estuvieran actualmente enseñando educación física en el quinto grado dentro de la educación primaria y que tuvieran comprobada experiencia impartiendo esta disciplina. Se contó con la participación de tres docentes, dos hombres y una mujer; cuentan con estudios a nivel posgrado, uno con maestría y otro con doctorado. Ambos docentes, además, cuentan con plazas de tiempo completo tanto en nivel básico como en nivel universitario. La tercera participante cursa estudios a nivel doctoral. La experiencia docente de los participantes en nivel básico es 12, 13 y 11 años respectivamente.

\section{Procedimiento}

Para el desarrollo de la presente investigación, y siguiendo los aspectos éticos, se solicitaron los permisos necesarios a los docentes, directores de escuelas y padres de familia a través de un consentimiento informado que tuvo que ser firmado antes de la implementación del proyecto, asimismo, antes de iniciar las grabaciones los estudiantes asintieron a participar en las mismas. Se previó instrumentar el procedimiento de la siguiente manera:

1. Diagnóstico. A través de un cuestionario de diagnóstico se buscó determinar los tipos de evaluación comúnmente utilizados por profesores de educación física en nivel primaria.

2. Programa de Formación. Se diseñó e implementó un programa de formación de profesores de educación física que proporcionó los elementos teórico-metodológicos que les facilitara construir proyectos formativos que integraran la evaluación auténtica, a fin de capacitarlos en función del diseño y ejecución de la evaluación auténtica de la docencia. En este programa de formación se desarrollaron secuencias didácticas e instrumentos que permitieran documentar las experiencias docentes.

3. Intervención. Se aplicaron las secuencias didácticas en un proceso de intervención en el aula por parte de los participantes, con la intención de valorar la aplicación de la evaluación auténtica por los profesores de educación física. En esta etapa se recolectó la información generada durante la intervención, se analizó el material y los 
George, E. B., \& González-Moreno, P. A. (2020). Evaluación auténtica como alternativa para la mejora en el proceso de enseñanza-aprendizaje de educación física en escuelas primarias. Revista Ciencias de la Actividad Física UCM, № 21(2), julio-diciembre, 1-19.

DOI: http://doi.org/10.29035/rcaf.21.2.3

datos obtenidos y los diversos registros recogidos en el proceso de la intervención (9 observacionesy 9 entrevistas, cada una de estas posterior a la observación; notas de campo).

4. Valoración de la experiencia. Se examinaron los logros alcanzados respecto de la planificación didáctica frente a lo logrado en la intervención con el uso de la evaluación auténtica en las clases de educación física, con el fin de valorar holísticamente la experiencia educativa.

\section{Secuencias Didácticas}

Las secuencias didácticas son el formato de planificación que los maestros utilizaron para la intervención en las clases con el quinto grado de primaria. De acuerdo con Marín \& Guzmán (2012), estas buscan el desarrollo de competencias desde un enfoque constructivista y una perspectiva sociocultural. Dichas secuencias didácticas apoyaron la incorporación de estrategias de evaluación auténtica, por lo que se diseñaron para facilitar los cambios en la forma en que los maestros evaluarían el desempeño de los estudiantes. Estas secuencias utilizaron situaciones problemáticas que brindaran a los estudiantes la oportunidad de resolverlos de manera auténtica o real, ayudándoles a ser innovadores y creativos durante el proceso.

Durante la intervención, las secuencias didácticas contemplaban que los estudiantes crearan una respuesta, generaran un producto o realizaran una demostración de la aplicación real del conocimiento obtenido. Los maestros utilizaron una variedad de instrumentos como observación, rúbricas y listas de cotejo para examinar el rendimiento en el aprendizaje, así como la calidad del proceso pedagógico.

\section{Instrumentos de recolección de datos}

En la presente investigación se emplearon tres tipos de estrategias para la recolección de datos: (1) El cuestionario en la fase de diagnóstico fue expresamente construido para este estudio con el fin de examinar los conocimientos previos de los docentes en materia de evaluación general y auténtica; este primer instrumento se aplicó al comienzo del taller y fue sujeto a revisión de expertos, para asegurar una adecuada credibilidad y confiabilidad de las preguntas, en función del objeto del estudio. (2) Observación participativa y estructurada durante el proceso de intervención en el aula (nueve observaciones, tres con cada participante; notas de campo); cada clase se grabó en video y también el investigador principal realizó diarios de campo sobre lo que había observado durante las actividades de la clase. Los temas utilizados durante la fase de observación del estudio fueron los siguientes: (a) el entorno de aprendizaje, que incluía las conductas y didáctica de los docentes, los materiales y el espacio disponible; y (b) la aplicación de las secuencias didácticas predefinidas, sus objetivos, los materiales didácticos considerados auténticos y la evaluación auténtica realizada. (3) Entrevistas semiestructuradas para indagar sobre el proceso de formación e intervención y la valoración de la experiencia por parte de los docentes; después de cada sesión observada (nueve en total), los profesores fueron entrevistados para obtener información potente sobre la intervención. Para la etapa de valoración de la experiencia, dos meses después de la intervención áulica, se realizó una entrevista adicional con cada uno de los participantes.

\section{Análisis de datos}

Para el análisis cualitativo de los datos se procedió a transcribir las observaciones y notas de campo realizadas durante la intervención, así como ingresar la información obtenida a 
George, E. B., \& González-Moreno, P. A. (2020). Evaluación auténtica como alternativa para la mejora en el proceso de enseñanza-aprendizaje de educación física en escuelas primarias. Revista Ciencias de la Actividad Física UCM, N²1(2), julio-diciembre, 1-19.

DOI: http://doi.org/10.29035/rcaf.21.2.3

través del cuestionario diagnóstico. Una vez realizado este proceso se utilizó el programa Atlas ti para la transcripción y posterior codificación de estos. Se revisaron las transcripciones y se envió una copia de cada una de ellas a cada participante para verificar que la información plasmada en ellas fuera precisa o se hiciera cualquier cambio si se consideraba necesario. La información se colocó en cuatro categorías diferentes: objetivo de la clase, material didáctico, ambiente de aprendizaje y proceso de evaluación, en este punto se utilizó la secuencia didáctica como elemento para hacer las distinciones de las categorías, después de lo cual la información se analizó en un orden secuencial.

Al final de cada sesión los profesores fueron entrevistados y grabados en video. Los videos con las entrevistas fueron revisados, transcritos y codificados, subsecuentemente, la información fue compartida con los diversos maestros para su verificación y aprobación. Después de analizar estas entrevistas, se observó que era necesario volver a entrevistar a los maestros, por lo tanto, el formato y el nivel de cuestionamiento cambiaron para aportar una mayor profundidad y calidad a la investigación. La información luego fue transcrita y reenviada a los maestros para su aprobación. Posteriormente se procedió a codificar dichas transcripciones. Para su análisis, las preguntas de las entrevistas se agruparon en cuatro categorías: opiniones sobre evaluación auténtica, taller de trabajo e intervención, ventajas y desventajas de la evaluación auténtica y problemas comunes en la educación física.

\section{Resultados}

En esta sección se muestran los resultados a los análisis de cuestionarios, observaciones y entrevistas realizadas. Estas categorías se seleccionaron con base en las preguntas de la investigación y han sido agrupados de acuerdo con cuatro temas principales: (a) tipos de evaluación comúnmente utilizados en educación física, (b) evaluación auténtica en educación física, (c) recursos y materiales auténticos en educación física y (d) planificación y retos en la educación física. El reporte del análisis cualitativo de los datos busca proporcionar una comprensión amplia de la evaluación auténtica en educación física en educación primaria, desde la perspectiva de los participantes en este estudio.

\section{Tipos de evaluación comúnmente uti- lizados en educación física}

En todas las áreas de la vida, el proceso de evaluación tiene lugar de una forma u otra. Si este proceso se elimina de la vida humana, tal vez se pierda su propósito. Es solo a través de la evaluación que uno puede discriminar entre bueno y malo, por lo que cada maestro debe estar preparado para usar varios tipos de evaluación para seleccionar el más apropiado para sus objetivos. En esta investigación, los docentes indicaron, mediante el cuestionario de diagnóstico, que tenían conocimiento sobre evaluaciones diagnósticas, sumativas, formativas, evaluación por competencia, continua, formal e informal. Sin embargo, en la primera entrevista cada uno indicó una tendencia a usar uno en particular; el maestro Hugo', señaló un mayor uso de la evaluación formativa, el maestro Matías ${ }^{2}$ la evaluación sumativa y la maestra Alejandra ${ }^{3}$, la evaluación continua.

De acuerdo con la información proporcionada en esa primera entrevista, la evaluación formativa utilizada por el maestro Hugo le proporcionaba información sobre la calidad de la instrucción y el progreso logrado en el aula. Además, él hacía uso de una prueba

ז,2,3 Pseudónimos 
George, E. B., \& González-Moreno, P. A. (2020). Evaluación auténtica como alternativa para la mejora en el proceso de enseñanza-aprendizaje de educación física en escuelas primarias. Revista Ciencias de la Actividad Física UCM, № 21(2), julio-diciembre, 1-19.

DOI: http://doi.org/10.29035/rcaf.21.2.3

diagnóstica al inicio del año escolar denominada Battelle diseñada por Newborg, Stock \& Wnek (1996) para identificar el nivel de sus estudiantes en cuanto a habilidades motrices básicas. Esta prueba examen califica la lateralidad, coordinación, espacio y tiempo de los estudiantes, luego de lo cual los resultados se recopilan y se colocan en un perfil. Sin embargo, el maestro no indicó si a estos resultados les daba seguimiento, por lo que es difícil establecer su relevancia en términos de evaluación dentro del ciclo escolar o rendimiento físico en los niños, ya que no se puede comparar cuanto han mejorado.

Por otra parte, la evaluación sumativa evaluación se utilizada por el maestro Matías la llevaba a cabo principalmente al final de la unidad de instrucción para obtener información sobre la medida en que los estudiantes dominaban el resultado de aprendizaje deseado y también para dar retroalimentación, adecuar sus objetivos y mejorar la efectividad en su instrucción. Además, incluía una competencia deportiva al comienzo del período escolar (futbol or baloncesto) para determinar el nivel de las habilidades y destrezas de los estudiantes, aprovechando los resultados para planificar el contenido y los objetivos para el final del ciclo o año escolar. Aunque esta estrategia puede considerarse limitada, pues un deporte resaltaría más las habilidades de algunos estudiantes respecto a otros, además podría limitar el interés de aquellos a los que el deporte seleccionado no le es de su agrado. En cuanto a la maestra Alejandra, aunque en la primera entrevista mencionó que la evaluación continua como principal medio para evaluar a sus estudiantes, no se observó evidencia física que corroborara dicha práctica.

Se pudo identificar que todos los maestros en el estudio utilizaron la observación como el instrumento principal para recopilar datos o información sobre sus alumnos en cuanto a evaluación se refiere. Del mismo modo, los maestros informaron en el cuestionario de diagnóstico que ellos tenían algún tipo de capacitación y conocimiento en evaluación, pero poca ayuda institucional en este aspecto específico de la evaluación. Todos los maestros pudieron dar una breve definición de lo que pensaban que es evaluación auténtica, sin embargo, solo el maestro Hugo había dicho inicialmente, en el cuestionario, que tenía cierta experiencia en evaluación auténtica.

\section{Evaluación auténtica en educación fí- sica}

Durante de la tercera entrevista, después de aplicadas las estrategias didácticas, los maestros reconocieron que la evaluación auténtica tiene otro enfoque de evaluación alternativa comparada con los tipos de evaluación tradicional o con frecuencia utilizada hasta antes de la intervención. Reconocieron que la capacitación en estrategias de evaluación auténtica fue oportuna, y reportaron que su aplicación específicamente fue beneficiosa para ellos y para los estudiantes de una forma $u$ otra. Los docentes resaltaron algunas de las características sobre la evaluación auténtica; para el maestro Matías, esta "es una evaluación diferente, más versátil, que procura una interacción más cercana entre maestro-estudiante, con recopilaciones más confiables y concretas".

La planificación de la clase fue fundamental para la aplicación de la evaluación auténtica durante la intervención, esta ayudó a conectar el contenido y métodos, así como seleccionar los más apropiados, preparar materiales que sean reales a las exigencias de los aprendizajes esperados, así como poder estimar el tiempo necesario para realizar las actividades y evaluar a los estudiantes a la misma vez.

Después de aplicadas las estrategias, el maestro Hugo comentó que "con la 
George, E. B., \& González-Moreno, P. A. (2020). Evaluación auténtica como alternativa para la mejora en el proceso de enseñanza-aprendizaje de educación física en escuelas primarias. Revista Ciencias de la Actividad Física UCM, № 21(2), julio-diciembre, 1-19.

DOI: http://doi.org/10.29035/rcaf.21.2.3

introducción de la evaluación auténtica en educación física sería posible cambiar la forma de enseñar y evaluar a los estudiantes en estas clases". Aquí observamos un cambio de perspectiva positivo, ya que este maestro antes del taller había indicado en una respuesta del cuestionario "no creo que tener un taller sobre evaluación auténtica sea útil para mí, porque he pasado cursos de evaluación auténtica durante un período en los Estados Unidos, en una institución superior". Los otros maestros concordaron que el conocimiento y las experiencias adquiridas durante el taller les ayudarían a aumentar la actividad física. Además, vieron que las estrategias auténticas deben pensarse detenidamente antes de realizar una evaluación. Para la maestra Alejandra, es necesario "proporcionar más preparación y talleres sobre cómo evaluar a los niños".

Cada maestro tenía su propia opinión sobre la evaluación auténtica y, además de los beneficios derivados de la evaluación auténtica, también reflexionaban sobre sus limitaciones; por ejemplo, el maestro Hugo creía que "Toma mucho tiempo," mientras que el maestro Matías argumentaba que la evaluación auténtica "es tediosa y lleva mucho tiempo debido a la gran cantidad de estudiantes que se evaluarán." Por su parte, la maestra Alejandra creía que, "Es mucho tiempo a planificar y ejecutar tanto la clase como la evaluación." Sin embargo, los maestros consideraban que los recursos auténticos ayudaban en la calidad de la enseñanza-aprendizaje y aportaban una nueva dinámica a la educación física ayudando a generar más interés y motivación en la participación en clase.

\section{Recursos y materiales auténticos en educación física}

Una de las tareas más desafiantes que los maestros de educación física atraviesan constantemente es cómo captar el interés de los estudiantes y desafiar su motivación para aprender. Los materiales del mundo real que los maestros de educación física traen al aula pueden contribuir al proceso general de aprendizaje de los estudiantes. Los maestros durante la tercera entrevista están fuertemente de acuerdo en que los recursos auténticos pueden usarse para enseñar y evaluar las habilidades, capacidades motrices básicas y el conocimiento de los estudiantes. Por lo tanto, después de que los maestros recibieron capacitación sobre el tema de las secuencias didácticas y la evaluación auténtica, durante la intervención se observó que los maestros usaron los siguientes recursos y materiales: aros, tarjetas, balones, bolos, cuerdas, conos, cajas, prefabricados, pizarra, escaleras y otras en sus clases. Hay que tomar en cuenta que cada recurso se utilizó de manera diferente en cada sesión, esto dependió del maestro y la actividad planificada.

Los maestros indicaron en la tercera entrevista que estos recursos y materiales ayudaron a alentar a los estudiantes a ser creativos (desarrollar una única interpretación o solución) permitiendo el trabajo independiente, resolviendo problemas y/o procesando información. También los utilizaron para verificar el nivel de comprensión en los puntos apropiados de la clase, promoviendo el interés de los alumnos en el contenido y motivando el aprendizaje. Coadyuvaron a vincular el conocimiento previo de los estudiantes con el resultado de aprendizaje esperado, les permitió construir vínculos significativos con actividades integradas del mundo real, lo cual es relevante para las necesidades de los estudiantes en general. Se utilizó de manera individual y grupal para el aprendizaje, y fomentaba la autoexpresión y la interacción del estudiante. Se observó que los recursos y materiales se usaron fácilmente en el espacio típicamente disponible, al evaluar las habilidades y capacidades motrices 
George, E. B., \& González-Moreno, P. A. (2020). Evaluación auténtica como alternativa para la mejora en el proceso de enseñanza-aprendizaje de educación física en escuelas primarias. Revista Ciencias de la Actividad Física UCM, N²1(2), julio-diciembre, 1-19.

DOI: http://doi.org/10.29035/rcaf.21.2.3

básicas. Los estudiantes podían usarlos para explicar, crear, demostrar y modificar actividades cuando los maestros lo consideraban necesario y relevante.

\section{Planificación didáctica}

Antes de la intervención los maestros describieron detalladamente el formato o planificación didáctica de sus clases de educación física, asimismo, el momento en que evaluarían a sus estudiantes. En cuanto a la planificación, todos coincidieron en la primera entrevista, que su clase constaba de tres fases (introducción, desarrollo y conclusión), y que los objetivos son seleccionados de acuerdo con el currículo que corresponda al grado escolar. Acerca de la evaluación solo un participante indicó realizar una evaluación diagnóstica al principio del año escolar, las otras evaluaciones dependían de cada maestro y de su obligación de tener una calificación trimestral para cada estudiante, sin contar con rúbricas ni guías estandarizadas para la asignatura. No obstante, en la última entrevista los maestros indicaron que las estrategias de evaluación auténtica los forzaron a poner mayor énfasis en el proceso de evaluación, planificar a través de listas de cotejos y rúbricas para cada clase de acuerdo con los objetivos planeados. Además, tener que dar a conocer a los estudiantes de donde provenía su calificación fueron puntos positivos que manifestaron los participantes al comparar el proceso de evaluación común con el uso de la evaluación auténtica.

Las estrategias no fueron complicadas y se evidenció el uso de diferentes tipos de instrumentos (e.g., lista de cotejos, observación directa entre otros) para evaluar el resultado del aprendizaje del alumno. De acuerdo con la maestra Alejandra3, "el propósito de la evaluación no es fallarle al niño sino observar y evaluar cuánto progreso ha logrado durante un cierto período de tiempo". A través de la evaluación auténtica, los maestros indicaron sentirse más confiados en la forma en que evaluaban a los estudiantes.

La practicidad de las estrategias de evaIuación auténtica estuvo directamente relacionada con la educación física al ser una disciplina mayormente práctica como lo es en México; es decir, las clases son prácticas y las actividades se centran en mejorar y desarrollar las habilidades motoras de los estudiantes, lo cual es importante y deseable para lograr un desarrollo integral del niño. Las actividades y juegos que se utilizaron en situaciones problemáticas alentaron a los niños a pensar y aplicar sus habilidades de aprendizaje. El análisis sugiere que las estrategias de evaluación auténtica implicaban un conocimiento aplicado, válido e innovador, en particular cuando se llevaban a cabo en un entorno real o cercano a un entorno real, lo que permitía a los estudiantes participar con entusiasmo.

\section{Retos en la educación física}

En la última entrevista realizada, los participantes indicaron que los problemas o barreras más comunes que enfrentan los maestros de educación física dentro del sistema escolar incluían los siguientes: (a) algunos directores y maestros de aula no ven la importancia de la educación física, por lo tanto, siempre existe alguna forma de resistencia cuando se trata de los estudiantes y su participación en este tipo de actividades escolares; (b) algunos padres no valoran la materia, y peor aún que sus hijos sean evaluados de manera negativa o incluso que tengan una calificación reprobatoria en esta disciplina. Además, los padres muestran más interés en las calificaciones de sus hijos en matemáticas, español, historia, geografía, en lugar de educación física; (c) la gran cantidad de estudiantes por grupo afecta la calidad del proceso de enseñanza aprendizaje por ende el proceso de evaluación también se ve afectado; 
George, E. B., \& González-Moreno, P. A. (2020). Evaluación auténtica como alternativa para la mejora en el proceso de enseñanza-aprendizaje de educación física en escuelas primarias. Revista Ciencias de la Actividad Física UCM, № 21(2), julio-diciembre, 1-19.

DOI: http://doi.org/10.29035/rcaf.21.2.3

(e) la ausencia de una guía de evaluación; y (f) el tiempo limitado para desarrollar una lección y evaluarla.

Otro reto, según la opinión del maestro Matías sobre la educación física era que "el programa de educación física en México se basa principalmente en las habilidades motoras y la corporeidad, que fueron aspectos de la década de 1940, por lo tanto, es necesario una reforma en la educación física, además de tener políticas sobre física educación dictada por la UNESCO y el Consejo Internacional de Salud y Educación Física y Deportes. Es hora de que comencemos a adoptar una educación física de calidad... es importante mencionar la educación física y cómo se puede mejorar". Además, también indicó "adaptando un plan de estudios razonable o nuevo y con la introducción de una evaluación auténtica al plan de estudios". También mencionó que "era bastante obvio los cambios radicales en el área de la educación física y los maestros están listos para aplicar estos cambios para mejorar la calidad de la educación física".

\section{Discusión}

Como se describió anteriormente, este estudio buscó examinar las estrategias, los instrumentos y los criterios de evaluación auténtica que los maestros de educación física aplicaban en sus clases de educación física de la escuela primaria, basándose en la planificación y aplicación de las secuencias didácticas. Los resultados de la investigación sugieren que los docentes tienen conocimiento de que existe una variedad en cuanto a tipos de evaluación se refiere, no obstante, podemos concluir que dicho conocimiento se ve limitado, ya que los maestros afirmaron conocerlos y aplicarlos, sin embargo, durante la entrevista se evidenció la confusión en cuanto al conocimiento teórico que dicen tener y la descripción de lo que dicen usar en la práctica. Por ejemplo, el profesor
Hugo señaló el uso de la evaluación formativa en sus clases de educación física, sin embargo, en lo que él describió no se reflejaba dicho tipo de evaluación, su respuesta puede deberse a que según los requisitos del currículo el proceso de enseñanza aprendizaje debe evaluarse de manera formativa, por tanto, es posible que los docentes solo indiquen su uso alejado de su práctica.

En este sentido, de acuerdo con LópezPastor \& Pérez-Pueyo (2017) la evaluación formativa "es todo proceso de evaluación cuya aplicación principal es mejorar los procesos de enseñanza-aprendizaje que tienen lugar, por lo que la mejora tendría que cumplir con una triple finalidad: (a) que el alumnado aprenda más, (b) que el profesorado mejore su práctica docente; y, (c) que mejore sobre la marcha el proceso de enseñanza-aprendizaje que lleva un cabo durante el trimestre, cuatrimestre o curso académico.

Aunque la evaluación formativa y auténtica están emparentadas, según Salom (2019) y López-Pastor \& Pérez-Pueyo (2017), la evaluación auténtica es ideal para la evaluación en educación física, ya que las técnicas e instrumentos pueden ser usados en situaciones, actividades y contenidos reales de aprendizaje. Salom (2019) también señala tres tipos de características que cumple la evaluación auténtica: está integrada en el proceso de enseñanza y aprendizaje, comparte el proceso de evaluación con el alumnado y da más importancia a una evaluación con una clara orientación formativa.

Al mismo tiempo, los maestros reconocieron el uso de la evaluación diagnóstica al inicio del período escolar con el propósito de ubicar a los estudiantes de manera apropiada al comienzo de la instrucción o descubrir las causas subyacentes de las deficiencias en el aprendizaje de los estudiantes como instrucción. Esta 
George, E. B., \& González-Moreno, P. A. (2020). Evaluación auténtica como alternativa para la mejora en el proceso de enseñanza-aprendizaje de educación física en escuelas primarias. Revista Ciencias de la Actividad Física UCM, N²1(2), julio-diciembre, 1-19.

DOI: http://doi.org/10.29035/rcaf.21.2.3

brinda información que alerta a los maestros sobre las fortalezas y debilidades de sus alumnos (Shim, Shakawi \& Azizan, 2017). Esta información se usa para mejorar su enseñanza y organizar un plan de instrucción sólido para facilitar el desarrollo educativo de los estudiantes (Sun \& Suzuki, 2013). También en la evaluación diagnóstica se obtiene información sobre el dominio de los estudiantes del conocimiento y las habilidades previas relevantes dentro del dominio, así como también revela las potencialidades y oportunidades para ajustar la enseñanza a estas características. El maestro Hugo realizaba una evaluación diagnóstica en dicho caso realizaba un test, pero no indicó que les diera seguimiento a los resultados recopilados o como estos influían en la planificación de sus clases.

A partir de su participación en el programa de formación en evaluación auténtica, los maestros creían que ésta les ayudaba a preparar lecciones reales, dinámicas y atractivas, y a satisfacer las necesidades de los estudiantes en función de sus habilidades, destrezas y conocimientos, como se ha reportado en investigaciones previas (Azri \& Al-Rashdi, 2014, Bazmi \& Ahmed, 2018, Castillo, Insuasty \& Osorio, 2017, Herod, 2002). Los maestros también creían que tenía un impacto positivo en el aprendizaje, a través de una variedad de mecanismos que ayudaban a mejorar el comportamiento, una mayor concentración y retención del aprendizaje mediante el uso de materiales o recursos auténticos utilizados en el desarrollo de sus clases. Los maestros indicaron que la evaluación auténtica motivaba a los estudiantes al permitirles ser parte del proceso de evaluación (Murphy, Fox, Freeman \& Hughes, 2017), estimulaba la participación (Brown, 2015), y ayudaba a mejorar las habilidades (Villarroel, Bloxham, Bruna, Bruna \& HerreraSeda, 2017). Además, consideraban que incluso estudiantes que tenían menos talento académico tenían la oportunidad de experimentar el éxito en un entorno de aprendizaje diferente en educación física.

Los maestros creían que a través de los procesos de evaluación auténtica ayudaban a los estudiantes a saber lo que estaban haciendo y proporcionaban comentarios positivos sobre cómo estaban progresando. En su opinión, la evaluación auténtica actuaba como motivador dándoles la oportunidad de evaluarse a sí mismos en un contexto práctico. Estos hallazgos se relacionan con los encontrados en las investigaciones de García-Jiménez, (2015), Nnorom \& Ukafor (2011) y Villarroel \& Bruna (2019), donde los docentes asumen el papel de facilitador y conciliador, manteniendo la retroalimentación necesaria para lograr una comprensión adecuada de los estudiantes y la estimulación efectiva de la construcción significativa del conocimiento.

Los tres maestros en el estudio declararon lo que entendieron sobre lo que es evaluación auténtica. El maestro Hugo lo entendió como una evaluación relacionada con la realidad social (Georgakis, Wilson \& Evans, 2015); el maestro Matías lo veía como una que indica la realidad de lo que se ha aprendido (Hay \& Penney, 2013) y la maestra Alejandra pensó que era igual a la realidad (Hay \& Penney, 2009, Mintah, 2003). Esto indica cuán eficiente y efectivamente pueden usar un repertorio de conocimientos y habilidades para negociar una tarea o actividad compleja, esto requiere que los maestros hagan juicios y sean innovadores. Otra ventaja es que los maestros les permiten a los estudiantes trabajar en grupos, dándoles oportunidades apropiadas para ensayar, practicar y consultar entre ellos y obtener retroalimentación y perfeccionar el rendimiento (ViIlarroel \& Bruna, 2019).

Los maestros, además, tuvieron oportunidad de evaluar si los estudiantes aplicaron las 
George, E. B., \& González-Moreno, P. A. (2020). Evaluación auténtica como alternativa para la mejora en el proceso de enseñanza-aprendizaje de educación física en escuelas primarias. Revista Ciencias de la Actividad Física UCM, N²1(2), julio-diciembre, 1-19.

DOI: http://doi.org/10.29035/rcaf.21.2.3

habilidades en una nueva situación. Los maestros pidieron a los estudiantes que hicieran su propia actividad en ese momento y evaluaron la capacidad de los estudiantes para interesarse, apropiarse o profundizar en su propio aprendizaje, y en diferentes momentos durante la lección, los maestros les permitieron a los estudiantes ser creativos y desarrollar ciertas actividades.

Los maestros de educación física en el estudio seguían el programa y la planificación de la Secretaría de Educación Pública (SEP) y sus planes eran supervisados constantemente, sin embargo, cada maestro utilizaba diferentes tipos de evaluación. Hay un poco de contradicción dentro de la SEP y otros cuerpos administrativos de educación y los maestros de educación física en relación con la evaluación en la educación física (INEP, 2018, Santiago, McGregor, Nusche, Ravela, \& Toledo, 2012), ya que no se cuenta con una evaluación disponible para evaluar los estudiantes de educación física. La única evaluación estandarizada a nivel nacional es la prueba ENLACE, que solo incluye contenidos de materias como matemáticas, español, geografía e historia, dejando fuera temas como la educación física (Pont, Figueroa, Zapata, Albiser \& Fraccola, 2013), por lo tanto, se deja todo proceso evaluativo a consideración de las escuelas y a los profesores de la asignatura.

Los maestros en este estudio creían firmemente que la educación física es una de las áreas que involucra el desarrollo de conocimientos, habilidades y actitudes a través del movimiento, y que es fundamental en el desarrollo de un estilo de vida activo y saludable durante toda la vida. A pesar de que esta disciplina tiene muy poco valor en el contexto mexicano, hay quienes creen que es importante para las condiciones físicas y de salud de los estudiantes de la nación, ya que ha sido señala como herramienta clave para reducir el problema de sobrepeso u obesidad en estudiantes (INSP, 2018). Por lo tanto, es importante que la educación física tenga el mismo valor que algunas de las otras asignaturas, aumente el número de horas y la frecuencia de la clase, y se estandaricen los procesos de enseñanza en la educación física con una evaluación que satisfaga el proceso de aprendizaje.

Como el valor educativo de la educación física parece ser poco comprendido entre los maestros de aula, los padres y los directores escolares, el desarrollo profesional debe centrarse en los objetivos clave de la educación física y en las estrategias para mejorar el éxito del programa. Los profesores del estudio indicaron que la evaluación auténtica es innovadora (Bazmi \& Ahmed, 2018), no complicada, real, entusiasta (Newman \& Wehlege, 1993), facilita un mayor aprendizaje (Artanayasa \& Giri, 2019), es motivadora (Mintah, 2003), y ofrece una gran variedad de instrumentos que pueden usarse para evaluar los resultados de aprendizaje de los estudiantes (Vallejo \& Molina, 2014). Los maestros pensaron que este estudio fue oportuno y apropiado y que se necesita más preparación y talleres en el área de evaluación. Sin embargo, es importante reconocer las limitaciones del estudio, la mayor dificultad de este proyecto fue la limitada participación de profesores de educación física, por lo cual, los resultados no pueden ser generalizados. No obstante, estos pueden ser un indicativo de que su utilización amerita ser considerada en la formación y práctica de profesores en formación.

\section{Conclusiones}

La intervención docente en las clases de educación física aquí evaluadas ha tenido muchas veces una postura evaluativa motivada más bien por cumplir con un requisito curricular y administrativo, así a través del taller se proporcionó una amplia gama de información y 
George, E. B., \& González-Moreno, P. A. (2020). Evaluación auténtica como alternativa para la mejora en el proceso de enseñanza-aprendizaje de educación física en escuelas primarias. Revista Ciencias de la Actividad Física UCM, N²1(2), julio-diciembre, 1-19.

DOI: http://doi.org/10.29035/rcaf.21.2.3

recursos en esta área, todavía existe la necesidad de brindar mayor preparación docente en el área pedagógica y de evaluación. Debido a la limitada participación docente no se pueden generalizar sus efectos positivos en el proceso de enseñanza y aprendizaje en educación física dentro de las escuelas primarias del estado de Chihuahua, por lo cual se sugiere realizar intervenciones y profundizar su estudio con muestras más grandes y longitudinales, incluir instrumentos que evalúen la perspectiva de los estudiantes acerca del proceso de evaluación en educación física.

Las estrategias de evaluación auténtica facilitaron el proceso de enseñanza y aprendizaje en las clases prácticas, ya que la retroalimentación fue clara, directa y breve, brindando a los estudiantes la oportunidad de aprender mediante una retroalimentación pertinente. Asimismo, el uso de materiales didácticos o recursos fue un factor importante en la enseñanza y evaluación de los estudiantes, este creó un ambiente adecuado para el desarrollo de los aspectos cognitivo y motriz.

Las estrategias promovieron la preparación anticipada de las clases, creación y utilización de materiales más reales y significativos para el logro de objetivos en el proceso de enseñanza aprendizaje. La evaluación auténtica es inclusiva en sus enfoques y puede ser útil para el progreso de los estudiantes, permitiéndoles comprender lo que tienen que hacer para progresar. La investigación confirma que los maestros consideran que la evaluación auténtica es dinámica, creativa, motivadora, innovadora y puede ayudar en el proceso de enseñanza y aprendizaje de los estudiantes en educación física. Contiene varios instrumentos que pueden ayudar a los maestros a ser creativos en la evaluación del aprendizaje de sus alumnos e innumerables recursos y materiales que pueden usarse como recursos de enseñanza, estimulando la participación y el aprendizaje en el aula. Sin embargo, el tiempo de instrucción para la educación física es de 100 minutos por semana y, en algunos casos, solo 50 minutos por semana para dedicarse a otras materias (especialmente matemáticas, español, ciencias o inglés) con la esperanza de lograr un mayor rendimiento académico. Sin embargo, varios estudios han identificado una relación significativa entre la actividad física y el rendimiento académico (Carlson et al., 2008, Rasberry, et al., 2011, Sallis et al., 1999). De acuerdo con SEP (2017) la educación física contribuye al desarrollo integral de un niño cuando desarrolla sus habilidades motoras y su corporalidad. La investigación también demuestra que la educación física no tiene ningún impacto negativo en el éxito de los estudiantes y que ofrece un estilo de vida saludable, gana y desarrolla una variedad de habilidades que los ayudan a lograr un estilo de vida activo. Los profesores del estudio pensaron que era oportuno, informativo y beneficioso para ellos como docentes y se necesitan más talleres enfocados en reforzar los conocimientos y habilidades en los procesos de evaluación. Por lo tanto, la evaluación auténtica representa una alternativa viable para mejorar la enseñanza y el aprendizaje de la educación física en la educación primaria. 
George, E. B., \& González-Moreno, P. A. (2020). Evaluación auténtica como alternativa para la mejora en el proceso de enseñanza-aprendizaje de educación física en escuelas primarias. Revista Ciencias de la Actividad Física UCM, N²1(2), julio-diciembre, 1-19.

DOI: http://doi.org/10.29035/rcaf.21.2.3

\section{REFERENCIAS BIBLIOGRÁFICAS}

Azri, R. H., \& Al-Rashdi, M. H. (2014). The effect of using authentic materials in teaching. International Journal of Scientific \& Technology Research, 3(10), 249-254. Recuperado de https://www.semanticscholar.org/paper/The-Effect-OfUsing-Authentic-Materials-In-Teaching-Azri-Al-

Rashdi/51f16706fd70899fd20ab3dc7f5 b021973c07200

Artanayasa, I. W., \& Giri, M. K. W. (2019). Learning models and authentic assessment on football skill learning achievement. International Journal of Physical Sciences and Engineering, 3(1), 22-31. DOI:

https://doi.org/10.29332/ijpse.v3n1.246

Bazmi, F. D., \& Ahmed, M. S. (2018). Impact of using authentic material on the academic achievement of secondary school students. Bodhi: International Journal of Research in Humanities, Arts and Science, 3(S2), 49-77. Recuperado de http://www.bodhijournals.com/pdf/sp I/December2018/Bodhi_V352.pdf

Brown, R. (2015). La evaluación auténtica: El uso de la evaluación para ayudar a los estudiantes a aprender. Relieve, Revista Electrónica de Investigación y EvaIuación Educativa, 27(2), 1-10. DOI: https://doi.org/10.7203/relieve.21.2.7674

Carlson, S. A., Fulton, J. E., Lee, S. M., Maynard, M., Brown, D. R., Kohl, H. W., \& Dietz, W. H. (2008). Physical education and academic achievement in elementary school: Data from the early childhood longitudinal study. American Journal of Public Health, 98(4), 721-727. DOI: $10.2105 / \mathrm{AJPH} .2007 .117176$
Castillo Losada, C. A., Insuasty, E. A., \& Osorio, M. F. (2017). The impact of authentic materials and tasks on students' communicative competence at a Colombian language school. Profile Issues in Teachers Professional Development, 19(1), 89-104. Recuperado de https://revistas.unal.edu.co/index.php/profile/article/view/56763/58142

García-Jiménez, E. (2015). La evaluación del aprendizaje: de la retroalimentación a la autorregulación. El papel de las tecnologías. Relieve. Revista Electrónica de Investigación y Evaluación Educativa, 27(2), 1-24.

DOI: https://doi.org/10.7203/relieve.21.2.7546

Georgakis, S., Wilson, R., \& Evans, J. (2015). Authentic assessment in physical education: A case study of game sense pedagogy. Physical Educator, 72(1), 67-86. Recuperado de https://eric.ed.gov/?id=EJ1059830

Gulikers, J. T., Bastiaens, T. J., y Kirschner, P. A. (2004). A five-dimensional framework for authentic assessment. Educational Technology Research and Development, 52(3), 67-86. Recuperado de https://research.ou.nl/en/publications/a-five-dimensional-frameworkfor-authentic-assessment

Hay, P., \& Penney, D. (2013). Assessment in physical education: A sociocultural perspective. New York, NY: Routledge.

Hay, P., \& Penney, D., (2009). Proposing in conditions for assessment efficacy in physical education. European Physical Review,15(3), 389-405. Recuperado de http://citeseerx.ist.psu.edu/viewdoc/download?doi=10.1.1.915.2077\&rep=rep1\&typ $e=p d f$ 
George, E. B., \& González-Moreno, P. A. (2020). Evaluación auténtica como alternativa para la mejora en el proceso de enseñanza-aprendizaje de educación física en escuelas primarias. Revista Ciencias de la Actividad Física UCM, N²1(2), julio-diciembre, 1-19.

DOI: http://doi.org/10.29035/rcaf.21.2.3

Herod, L. (2002). Adult learning from theory to practice. Recuperado de http://jarche.com/wp-content/uploads/2015/12/adult_learning.pdf

Instituto Nacional de Salud Pública (México) (2018). Hacia una estrategia nacional para la prestación de educación física de calidad en el nivel básico del sistema educativo mexicano. Recuperado de https://www.insp.mx/produccion-editorial/novedades-editoriales/4974-estrategia-presentar-educacion-fisica-nivel-basico.html

Jabbarifar, T. (2009). The importance of classroom assessment and evaluation in educational system. Trabajo presentado en 2nd International Conference of Teaching and Learning (pp. 1-9). INTI University College, Malaysia. Recuperado de https://pdfs.semanticscho-

lar.org/db8c/4d3e5e56aa80c220e17e eac25183acaaa43d.pdf

Karim, A. A., Abduh, A., Manda, D., \& Yunus, M. (2018). The effectivity of authentic assessment-based character education evaluation model. TEM Journal, 7(3), 495-500. Recuperado de http://www.temjournal.com/content/73/TemJournalAugust2018_495_500.pdf

López-Pastor, V., \& Pérez-Pueyo, Á. (Eds.). (2017). Buenas prácticas docentes. Evaluación formativa y compartida en educación: Experiencias de éxito en todas las etapas educativas. León: Universidad de León.

López-Taylor, J., Jáuregui-Ulloa, E., \& GonzálezVillalobos, M. (2013). Physical education in Mexico: Experiences and trends related with physical activity and health. En M. K. Chin \& C. R. Edginton (Eds.), Physical education and health: Global perspectives and best practices (pp. 311-324). Champaign, IL: Sagamore Publishing LLC.

Marín, R. \& Guzmán, I., (2012). Formación-evaluación: una propuesta para el desarrollo y evaluación de competencias docentes. En E. Cisneros Chacón, B. García Cabrero, E. Luna \& R. Marín Uribe (Coords.), Evaluación de competencias docentes en la educación superior, (pp. 203-247), Ciudad de México: Juan Pablos Editor.

Mertens, D. M. (2015). Research and evaluation in education and psychology: Integrating diversity with quantitative, qualitative, and mixed methods. Thousand Oaks, CA: Sage.

Mintah, J. K. (2003). Authentic assessment in physical education: Prevalence of use and perceived impact on students' self-concept, motivation, and skill achievement. Measurement in Physical Education and Exercise Science, 7(3), 161-174. DOI: https://doi.org/10.1207/S15327841MPE E0703_03

Molina-Luque, F. (2018). Educación física, calidad de vida y la nueva sociología de la infancia: Repensando la metodología mixta en sociología. Retos: Nuevas Tendencias en Educación Física, Deporte y Recreación, (33), 69-73. Recuperado de https://recyt.fecyt.es/index.php/retos/article/view/51510

Moreno, O. T. (2012). La evaluación de competencias en educación. Sinéctica, Revista Electrónica de Educación, (39), 120. Recuperado de http://www.scielo.org.mx/scielo.php?s 
George, E. B., \& González-Moreno, P. A. (2020). Evaluación auténtica como alternativa para la mejora en el proceso de enseñanza-aprendizaje de educación física en escuelas primarias. Revista Ciencias de la Actividad Física UCM, № 21(2), julio-diciembre, 1-19.

DOI: http://doi.org/10.29035/rcaf.21.2.3

cript=sci_arttext\&pid=S1665-

$109 \times 2012000200010$

Murphy, V., Fox, J., Freeman, S., \& Hughes, N. (2017). "Keeping it Real": A review of the benefits, challenges and steps towards implementing authentic assessment. AISHE-J: The All Ireland Journal of Teaching and Learning in Higher Education, 9(3), 3231-32313. Recuperado de

https://ojs.aishe.org/aishe/index.php/aishe-j/article/view/280

Newborg, J., Stock, J. R., \& Wnek, L. (1996). Battelle: Inventario de desarrollo. Madrid: TEA Ediciones.

Newman, F. M., \& Wehlage, G. G. (1993). Five standards of authentic instruction. Educational Leadership, 50(7), 8-12. Recuperado de http://www.ascd.org/publications/educational-leader-

ship/apr93/vol50/num07/Five-Standards-of-Authentic-Instruction.aspx

Nnorom, N., \& Ukafor, T. (2011). Authentic assessment method in science: Study of issues for teachers. ANSU Journal of Integrated Knowledge, 7(1), 202-209. Recuperado de https://www.academia.edu/38896467/AUTHEN-

TIC_ASSESSMENT_METHOD_IN_SCI-

ENCE_STUDY_OF_ISSUES_FOR_TEACHERS

Osborne, R., Belmont, R. S., Peixoto, R. P., Azevedo, I. O. S. D., \& Carvalho Junior, A. F. P. D. (2016). Obstacles for physical education teachers in public schools: An unsustainable situation. Motriz: Revista de Educação Física, 22(4), 310-318.

DOI:

https://doi.org/10.1590/s1980-

6574201600040015
Pont, B., Figueroa, D. T., Zapata, J., Albiser, E., \& Fraccola, S. (2013). Education policy outlook: Mexico. OECD Publishing. Recuperado de http://www.oecd.org/mexico/EDUCA TION\%2OPOLICY\%20OUTLOOK\%2OM EXICO_EN.pdf

Rasberry, C. N., Lee, S. M., Robin, L., Laris, B. A., Rusell, L. A., Coyle, K. K., \& Nihiser, A. J. (2011). The association between school-based physical activity, including physical education, and academic performance: A systematic review of the literature. Preventive Medicine, 52, S10-S20. Recuperado de https://www.sciencedirect.com/science/article/abs/pii/s0091743511000557?via\%3 Dihub

Ramírez G., A. A., \& Chel H., D. E. (2019). Análisis de la Reforma Educativa en México, desde la perspectiva de Educación Física. Revista Ciencias de la Actividad Física UCM, 20(2), 1-17. DOI: https://doi.org/10.29035/rcaf.20.2.2

Sallis, J. F., McKenzie, T. L., Kolody, B., Lewis, M., Marshall, S., \& Rosengard, P. (1999). Effects of health-related physical education on academic achievement: Project SPARK. Research Quarterly for Exercise and Sport, 70(2), 127-134. Recuperado de https://www.tandfonline.com/doi/abs /10.1080/02701367.1999.10608030

Salom, M. A. C. (2019). Una oportunidad para avanzar hacia la evaluación auténtica en Educación Física. Retos: nuevas tendencias en educación física, deportey recreación, (36), 259-265. Recuperado de https://recyt.fecyt.es/index.php/retos/article/view/67540 
George, E. B., \& González-Moreno, P. A. (2020). Evaluación auténtica como alternativa para la mejora en el proceso de enseñanza-aprendizaje de educación física en escuelas primarias. Revista Ciencias de la Actividad Física UCM, № 21(2), julio-diciembre, 1-19.

DOI: http://doi.org/10.29035/rcaf.21.2.3

Santiago, P., McGregor, I., Nusche, D., Ravela, P., \& Toledo, D. (2012). OECD reviews of evaluation and assessment in education: Mexico 2012. OECD Publishing. Recuperado de

https://www.oecd.org/mexico/Mexico\%20Review\%20of\%20Evaluation\%20and\%20Assessment\%20in\%20Education.pdf

Secretaría de Educación Pública (SEP). (2017). Aprendizajes clave para la educación integral. Ciudad de México: Secretaría de Educación Pública. Recuperado de https://www.tamaulipas.gob.mx/educacion/wp-content/uploads/sites/3/2017/07/aprendizajes_clave_para_la_educacion_integral.pdf

Secretaría de Educación Pública. (2019). Guía de trabajo para el Taller de capacitación "Hacia una Nueva Escuela Mexicana". Secretaria de Educación Pública: México. Recuperado de https://es.slideshare.net/ma072001/g ua-de-trabajo-para-el-taller-decapacitacin-hacia-una-nuevaescuela-mexicana-ciclo-escolar20192020

Shim, G. T. G., Shakawi, A. M. H. A., \& Azizan, F. L. (2017). Relationship between students' diagnostic assessment and achievement in a pre-university mathematics Course. Journal of Education and Learning, 6(4), 364-371. Recuperado de http://www.ccsenet.org/journal/index.php/jel/article/view/69209

Sun, Y., \& Suzuki, M. (2013). Diagnostic assessment for improving teaching practice. International Journal of Information and Education Technology,
3(6), 607-610. Recuperado de http://www.ijiet.org/papers/345-

T021.pdf

Vallejo Ruiz, M., \& Molina Saorín, J. (2014). La evaluación auténtica de los procesos educativos. Revista Iberoamericana de Educación, 64, 11-25. Recuperado de https://rieoei.org/RIE/article/view/403

Villarroel, V., \& Bruna, D. (2019). ¿Evaluamos lo que realmente importa? el desafío de la evaluación auténtica en educación superior. Revista Calidad en la Educación, 50, 492-509. Recuperado de https://www.calidadenlaeducacion.cl/index.php/rce/article/view/729

Villarroel, V., Bloxham, S., Bruna, D., Bruna, C., \& Herrera-Seda, C. (2017). Authentic assessment: Creating a blueprint for course design. Assessment \& Evaluation in Higher Education, 43(5), 840854.

DOI: https://doi.org/10.1080/02602938.2017. 1412396

Yin, R. K. (2018). Case study research and applications: Design and methods. Thousand Oaks, CA: Sage.

Nota: Proyecto financiado por el Consejo $\mathrm{Na}$ cional de Ciencia y Tecnología (CONACYT), a través del Programa Nacional de posgrados de Calidad (PNPC) 
George, E. B., \& González-Moreno, P. A. (2020). Evaluación auténtica como alternativa para la mejora en el proceso de enseñanza-aprendizaje de educación física en escuelas primarias. Revista Ciencias de la Actividad Física UCM, № 21(2), julio-diciembre, 1-19.

DOI: http://doi.org/10.29035/rcaf.21.2.3

\section{Dirección para correspondencia}

Eversley Bernard George

Universidad Autónoma de Chihuahua

Chihuahua, México

Dirección postal: Ruta de las Humanidades S/N,

Campus Universitario 1, Av. Universidad S/N

ORCID: https://orcid.org/0000-0003-3118-1505

Contacto:

bernard_george777@hotmail.com

Recibido: 23-05-2020

Aceptado: 11-08-2020

\section{(ㄷ) (1) (2)}

Esta obra está bajo una Licencia de Creative Commons Reconocimiento-NoComercialCompartirlgual 4.0 Internacional. 